\title{
DISTORTED-WAVE BORN AND DISTORTED-WAVE RYTOV APPROXIMATIONS
}

\author{
G. BEYLKIN and M.L. ORISTAGLIO \\ Schlumberger-Doll Research, Ridgefield, CT 06877-4108, USA
}

Received 4 December 1984

\begin{abstract}
The relation is considered between the distorted-wave Born (DWB) and the distorted-wave Rytov (DWR) approximations. Analyzing the Helmholtz equation, it is shown that the formal asymptotic justification of DWB and DWR approximations remains the same as that of the ordinary ones. A relation is derived between the first DWB and DWR approximations and an example given to emphasize that these approximations, though simply related, have quite different ranges of accuracy.
\end{abstract}

This paper considers the relation between the distorted-wave Born (DWB) and the distorted-wave Rytov (DWR) approximations. The ordinary Born [1] and Rytov [2] approximations are used to simplify both forward and inverse problems of wave propagation in applications ranging from nuclear physics to seismic exploration (see refs. [4-7], for example). Within these approximations, the solution of a partial differential equation is expressed as a perturbation about a known solution to a simpler equation. The only difference between the ordinary and distorted-wave approximations is that for the distorted-wave approach, one assumes that the known solution is already "perturbed" relative to some ideal, simple model.

To illustrate this we consider the Helmholtz equation and show that the formal asymptotic justification of DWB and DWR approximations remains the same as that of the ordinary ones [3]. We also derive a relation between the first DWB and DWR approximations and give an example to show that these approximations, though simply related, have quite different ranges of accuracy.

We start with the homogeneous Helmholtz equation

$\left[\nabla^{2}+k^{2} n^{2}(x)\right] U(x, k)=0$,

where $n(x)$ is the index of refraction. We assume that

$n^{2}(x)=n_{0}^{2}(x)+\epsilon n_{1}(x)+\epsilon^{2} n_{2}(x)+\ldots$,

where $\epsilon$ is a small parainieter. The index of refraction $n_{0}(x)$ corresponds to the unperturbed model. In the case of the ordinary Born and Rytov approximations $n_{0}(x)$ is a constant.

The DWB approximation can be formally obtained if we seek a solution of eq. (1) in the form

$U(x, k)=U_{0}(x, k)+\epsilon U_{1}(x, k)+\ldots$

Substituting expression (3) in (1) and equating coefficients of like powers of $\epsilon$, we arrive at equations for the functions $U_{j}(x, k), j=0,1 \ldots$ :

$\left(\nabla^{2}+k^{2} n_{0}^{2}\right) U_{0}=0$,

$\left(\nabla^{2}+k^{2} n_{0}^{2}\right) U_{1}=-k^{2} n_{1} U_{0}$,

$\left(\nabla^{2}+k^{2} n_{0}^{2}\right) U_{2}=-k^{2} n_{2} U_{0}-k^{2} n_{1} U_{1}$,

-...

Eq. (3) is the DWB approximation and eqs. (4) show how to compute consecutive terms of series for $U$.

In particular, if $n_{0}^{2}(x)=1$, then the DWB approximation reduces to the classical Bron approximation. We then have $U_{0}^{ \pm}(x, k)=\exp ( \pm \mathrm{i} k x \cdot v)$, where $v$ is a unit vector, and the function $U_{1}(x, z)$ satisfies the equation $\left(\nabla^{2}+k^{2}\right) U_{1}(x, k)=-k^{2} n_{1}(x) \exp ( \pm \mathrm{i} k x \cdot v)$,

etc. (see ref. [4] for example).

We turn now to the Rytov approximation. The DWR 
approximation can be obtained if we seek a solution of eq. (1) in the form

$U(x, k)=\mathrm{e}^{\mathrm{i} k \Phi(x, k)}$,

where the phase function $\Phi(x, k)$ is a formal series

$\Phi(x, k)=\Phi_{0}(x, k)+\epsilon \Phi_{1}(x, k)+\epsilon^{2} \Phi_{2}(x, k)+\ldots$.

Using (5) and (1) we find that the phase function $\Phi(x, k)$ satisfies the equation

$(\nabla \Phi)^{2}-n^{2}+(1 / i k) \nabla^{2} \Phi=0$.

We now substitute the series (6) in (7), equate the coefficients of powers of $\epsilon$, and arrive at equations for functions $\Phi_{j}(x, k), j=0,1 \ldots$ :

$\left(\nabla \Phi_{0}\right)^{2}+(1 / \mathrm{i} k) \nabla^{2} \Phi_{0}-n_{0}^{2}=0$

$2 \nabla \Phi_{0} \cdot \nabla \Phi_{1}+(1 / \mathrm{i} k) \nabla^{2} \Phi_{1}-n_{1}=0$,

$2 \nabla \Phi_{0} \cdot \nabla \Phi_{2}+(1 / \mathrm{i} k) \nabla^{2} \Phi_{2}-n_{2}+\left(\nabla \Phi_{1}\right)^{2}=0$

Eqs. (5) and (6) are the DWR approximation and eqs.

(8) show how to compute the consecutive terms of the series for $\Phi$. Let us now compare DWB and DWR approximations. It is easy to estimate the relative error of the $m$ th DWR approximation. Indeed, it follows from (5) and (6) that

$$
\begin{aligned}
& \left(U-U_{\mathrm{R}}^{m}\right) / U=1-\exp \left(-\mathrm{i} k \sum_{j=m+1}^{\infty} e^{j} \Phi_{j}\right) \\
& =O\left(\mathrm{i} k \epsilon^{m+1} \Phi_{m+1}\right),
\end{aligned}
$$

where $U_{\mathrm{R}}^{m}$ is the $m$ th Rytov approximation,

$U_{\mathrm{R}}^{m}(x, k)=\exp \left(\mathrm{i} k \sum_{j=0}^{m} \epsilon^{j} \Phi_{j}(x, k)\right)$.

To estimate the relative error of the DWB approximation we first establish relations between terms in series in (6) and (3). We have

$$
\begin{aligned}
& U(x, k)=\mathrm{e}^{\mathrm{i} k \Phi_{0}} \sum_{d=0}^{\infty} \frac{1}{d !}\left(\mathrm{i} k \sum_{j=1}^{\infty} \epsilon^{j} \Phi_{j}\right)^{d} \\
& =\mathrm{e}^{\mathrm{i} k \Phi_{0}} \sum_{l=0}^{m} \epsilon^{l} \sum_{d=0}^{l} \frac{(\mathrm{i} k)^{d}}{d !} \\
& \times \sum_{j_{1}+j_{2}+\ldots+j_{d}=1} \Phi_{j_{1}} \Phi_{j_{2}} \ldots \Phi_{j_{d}} .
\end{aligned}
$$

The $m$ th DWB approximation is the sum of the $m+1$ first terms in (10),

$U_{\mathrm{B}}^{m}=\mathrm{e}^{\mathrm{i} k \Phi_{0}} \sum_{l=0}^{m} c^{l} \sum_{d=0}^{l} \frac{(\mathrm{i} k)^{d}}{d !} \sum_{j_{1}+j_{2}+\ldots+j_{d}=l} \Phi_{j_{1}} \Phi_{j_{2}} \ldots \Phi_{j_{d}}$.

Thereby, we have

$$
\begin{gathered}
\left(U-U_{\mathrm{B}}^{m}\right) / U=O\left(\epsilon^{m+1} \sum_{d=0}^{m+1} \frac{(\mathrm{i} k)^{d}}{d !}\right. \\
\left.\times \sum_{j_{1}+j_{2}+\ldots+j_{d}=m+1} \Phi_{j_{1}} \Phi_{j_{2}} \ldots \Phi_{j_{d}}\right)
\end{gathered}
$$

Specifying the estimates (9) and (11) to the first DWB and DWR approximations, we have

$$
\begin{aligned}
(U & \left.-U_{\mathrm{R}}^{1}\right) / U=1-\exp \left(-\mathrm{i} k \sum_{j=2}^{\infty} \epsilon^{j} \Phi_{j}\right) \\
& =O\left(\mathrm{i} k \epsilon^{2} \Phi_{2}\right),
\end{aligned}
$$

and

$\left(U-U_{\mathrm{B}}^{1}\right) / U=\overline{\mathcal{O}}\left(\epsilon^{2}\left(\mathrm{i} k \Phi_{2}-\frac{1}{2} k^{2} \Phi_{1}^{2}\right)\right)$.

When $x$ and $k$ are fixed, estimates in (9) and (11) demonstrate that both DWB and DWR approximations are of the same order of accuracy with respect to $\epsilon$. Clearly, however, the errors in these two approximations will behave differently as functions of $x$ and $k$.

Let us consider now the relation between the first DWB and the first DWR approximations. This relation for ordinary Born and Rytov approximations is of importance in linearized inverse scattering problems [7]. We set

$\Phi_{1}=\mathrm{e}^{-\mathrm{i} k \Phi_{0}} W_{1}$

and obtain from (8) that the function $W_{1}$ satisfies the 
following equation

$\left[\nabla^{2}+k^{2} n_{0}^{2}(x)\right] W_{1}=\mathrm{i} k n_{1} \mathrm{e}^{\mathrm{i} k \Phi_{0}}$.

Also, from expressions (6) and (12) we have

$\Phi_{\mathrm{R}}^{1}=\Phi_{0}+\epsilon \mathrm{e}^{-\mathrm{i} k \Phi_{0}} W_{1}$.

Comparing (13) and the equation for the function $U_{1}$ in (4) and using (14) we arrive at the relation between the first DWB and DWR approximations,

$\Phi_{\mathrm{R}}^{1}=\Phi_{0}+(\epsilon / \mathrm{i} k) \mathrm{e}^{-\mathrm{i} k \Phi_{0}} U_{1}$,

where $U_{1}$ is the first-order term in the DWB approximation,

$U_{\mathrm{B}}^{1}=U_{0}+\epsilon U_{1}$.

If $n_{0}(x)=1$, relation (15) reduces to the well-known relation between classical Born and Rytov approximations [8].

The first DWR and DWB approximations are always related through (15), but the domains over which they are accurate can be quite different as follows from estimates in (9a) and (11a). To show this, we provide a simple example. Since DWR and DWB do not differ from ordinary Rytov and Born approximations with respect to this property, our example deals with the ordinary ones for simplicity.

Consider a plane wave incident upon the interface between two homogeneous halfspaces (fig. 1). Let $(y, z)$

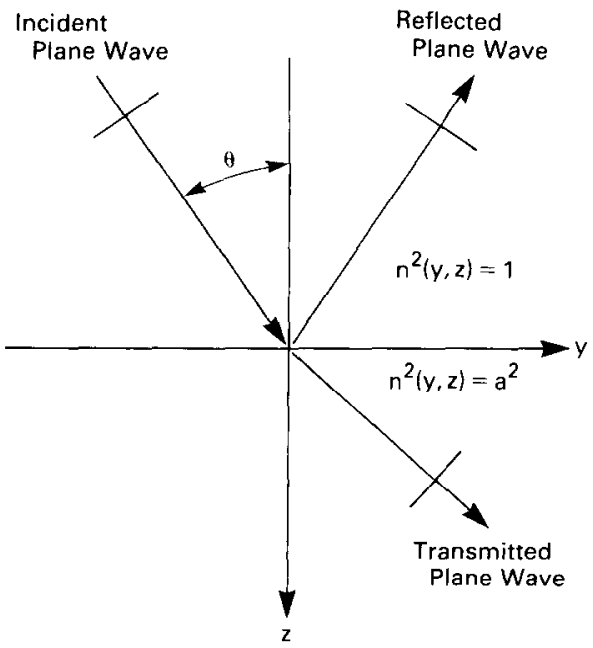

Fig. 1. Planc wave incident upon tne anterface between two homogeneous halfspaces. be coordinates of points in this space and let the index of refraction be as follows

$n^{2}(y, z)=1+n_{1}(y, z)$,

where

$$
\begin{array}{rlrl}
n_{1}(y, z) \equiv n_{1}(z) & =0, & & z<0 ; \\
& =a^{2}-1, \quad z>0
\end{array}
$$

and $a$ is a posilive constant. Comparing (16) with (2) we set the parameter $\epsilon=1$ in further consideration.

The problem of scattering of the plane wave $\exp [\mathrm{i} k(y \sin \theta+z \cos \theta)]$,

where $\theta$ is a fixed angle and $k$ is the wave number, can be solved explicitly. We have the following expressions for the field

$$
\begin{array}{rlr}
u(y, z)= & \exp [\mathrm{i} k(y \sin \theta+z \cos \theta)] \\
& +R \exp [\mathrm{i} k(y \sin \theta-z \cos \theta)], \quad z<0 ; \\
= & T \exp \left\{\mathrm{i} k\left[y \sin \theta+z(1+\alpha)^{1 / 2} \cos \theta\right]\right\}, z>0,
\end{array}
$$

where

$\alpha=\left(a^{2}-1\right) / \cos ^{2} \theta$.

The reflection and transmission coefficients are given respectively by

$R=\frac{1-(1+\alpha)^{1 / 2}}{1+(1+\alpha)^{1 / 2}}, \quad T=\frac{2}{1+(1+\alpha)^{1 / 2}}$.

To obtain the Rytov approximation to the field in (18) using a constant background with the index of refraction $n_{0}^{2}=1$ we first compute the phase of the background field. The phase of the background field is the phase of the plane wave which is as follows

$\Phi_{0}=y \sin \theta+z \cos \theta$.

The first perturbation of the phase, the function $\Phi_{1}$, depends only on $z$ and satisfies the corresponding equation in (8) which in this case reduces to

$2 \cos \theta \frac{\mathrm{d} \Phi_{1}(z)}{\mathrm{d} z}+\frac{1}{\mathrm{i} k} \frac{\mathrm{d}^{2} \Phi_{1}(z)}{\mathrm{d} z^{2}}=n_{1}(z)$,

where $n_{1}(z)$ is described in (17). $\Phi_{1}(z)$ and its normal derivative $\mathrm{d} \Phi_{1}(z) / \mathrm{d} z$ should be continuous at $z=0$. Using these continuity conditions together with the 
condition for the field to be outgoing for $z>0$ we solve (19) and arrive at

$$
\begin{aligned}
\Phi_{1}(y, z) \equiv \Phi_{1}(z) & \\
=-(\alpha / 4 \mathrm{i} k) \exp (-2 \mathrm{i} k z \cos \theta), & z<0 ; \\
=(z \alpha / 2) \cos \theta-\alpha / 4 \mathrm{i} k, & z>0 .
\end{aligned}
$$

Therefore, the first Rytov approximation to the field is a follows

$$
\begin{aligned}
u^{\mathrm{K}}(y, z)= & \exp [\mathrm{i} k(y \sin \theta+z \cos \theta) \\
& \left.-\frac{1}{4} \alpha \mathrm{e}^{-2 \mathrm{i} k z \cos \theta}\right], \quad z<0 ; \\
= & \exp [\mathrm{i} k(y \sin \theta+z \cos \theta) \\
& \left.+\frac{1}{2} \mathrm{i} k z \alpha \cos \theta-\frac{1}{4} \alpha\right], \quad z>0 .
\end{aligned}
$$

Similar considerations of eq. (4) for the first Born approximation yield

$$
\begin{array}{rlrl}
u^{\mathrm{B}}(y, z)= & \exp [\mathrm{i} k(y \sin \theta+z \cos \theta)] \\
& -\frac{1}{4} \alpha \exp [\mathrm{i} k(y \sin \theta-z \cos \theta)], & & z<0 ; \\
= & {\left[1-\frac{1}{4} \alpha(1-2 \mathrm{i} k z \cos \theta)\right]} & \\
& \times \exp [\mathrm{i} k(y \sin \theta+z \cos \theta)], & & z>0 .
\end{array}
$$

Eqs. (21) and (22) are obviously related through (15). However, for a given value of $z$, their accuracy is quite different, as follows from comparisons with the exact solution. In particular, the difference between the two is dramatic for the transmitted field $(z>0)$ due to the presence of the term proportional to $z$ in the Born approximation (22) for the transmitted field. Because of this term, the error accumulates with increasing $z$, no matter how small the perturbation is. In contrast, the
Kytov approximation (21) provides a reasonable answer.

The same conclusion about the behavior of Born and Rytov approximations can be drawn from estimates (9a) and (11a). Using corresponding equation in (8) we compute the function $\Phi_{2}$ and obtain

$$
\begin{array}{rlrl}
\mathrm{i} k \Phi_{2}(z)= & \frac{1}{8} \alpha^{2} \exp (-2 \mathrm{i} k z \cos \theta) \\
& -\frac{1}{32} \alpha^{2} \exp (-4 \mathrm{i} k z \cos \theta), \quad & & z<0 ; \\
= & -\frac{1}{8} \mathrm{i} k z \alpha^{2} \cos \theta+\frac{3}{32} \alpha^{2}, & & z>0 .
\end{array}
$$

Compared to the relative error of the Rytov approximation (9a), the estimate of the relative error of the Born approximation (11a) has an extra term $\frac{1}{2} k^{2} \Phi_{1}^{2}$. It follows from (20) that this term is as follows

$$
\begin{aligned}
\frac{1}{2} k^{2} \Phi_{1}^{2}(z) & =-\frac{1}{32} \alpha^{2}, & & z<0 ; \\
& =\frac{1}{8} \alpha^{2}(k z \cos \theta-1 / 2 \mathrm{i})^{2}, & & z>0,
\end{aligned}
$$

which predicts much faster accumulation of error in the Born approximation compared to the Rytov approximation for the transmitted field $(z>0)$.

\section{References}

[1] M. Born, Z. Physik 38 (1926) 803.

[2] S.M. Rytov, Izv. Akad. Nauk SSSR Ser. Fiz. 2 (1937) 223.

[3] J.B. Keller, J. Opt. Soc. Am. 59 (1969) 1003.

[4] J.R. Taylor, Scattering theory (Wiley, New York, 1972).

[5] V.I. Tatarski, Wave propagation in turbulent medium (McGraw-Hill, New York, 1961).

[6] J.K. Cohen and N. Bleistein, Geophysics 44 (1979) 1077.

[7] A.J. Devaney, Ultrasonic Imaging 4 (1982) 336.

[8] A. Nayfeh, Perturbation methods (Wiley, New York, 1973). 